\title{
CROSS-POSITIVE MATRICES*
}

\author{
HANS SCHNEIDER + AND MATHUKUMALLI VIDYASAGAR
}

Dedicated 10 Alston Householder on the occasion of his 65 th hirlhdav.

1. Introduction. In recent years there has been a great deal of interest in a matrix $A$ which is positive on a cone $C$ in Euclidean $n$-space, i.e., $A C \leqq C$ (e.g., Birkhoff [2] and Vandergraft [5]). Another type of positivity is considered by Haynsworth and Hoffman [4] for symmetric $A$ and self-polar $C$.

In this paper (\$3) we introduce three classes of matrices related to the class of positive matrices: the class of cross-positive matrices on $C$, strongly cross-positive on $C$, and strictly cross-positive on $C$. These classes contain respectively extensions, by multiples of the identity matrix, of the class of matrices positive on $C$, irreducible on $C$, and strictly positive on $C$. In this section we also investigate when equality occurs in the various containment relations. In $\S 4$ we consider exponentials of cross-positive matrices. Then $(\$ 5)$ we prove theorems of Perron-Frobenius type for each class of cross-positive matrices. Thus in the case of some cones $C$, we obtain extensions of the standard Perron-Frobenius theorems. Sections 6 and 7 are devoted to matrices cross-positive on a polyhedral cone and symmetric crosspositive matrices, respectively. We state some open problems in $\S 8$. We begin by assembling in $\S 2$ some preliminary lemmas on cones in a form in which they are used in this paper.

\section{Lemmas on cones.}

Defintion 1. A set $C$ in real Euclidean $n$-space $R^{n}$ is said to be a cone if

(i) $C$ is nonempty,

(ii) $C$ is a closed subset of $R^{n}$,

(iii) $C+C \subseteq C$,

(iv) $\alpha C \subseteq C$ for all $\alpha>0$,

(v) $C-C=R^{n}$,

(vi) $C \cap(-C)=\{0\}$.

It should be observed that many authors employ the term "cone" for subsets of $R^{n}$ satisfying some, but not all, of the above conditions.

We shall denote the inner product in $R^{n}$ by $(z, y)=z^{T} y$ and we write $\|z\|^{2}$ $=(z, z),\|z\| \geqq 0$.

DefintTiOn 2. The polar $S^{*}$ of a nonempty set $S$ in $R^{n}$ is defined to be

$$
S^{*}=\left\{z \in R^{n}:(z, y) \geqq 0 \text { for all } y \in S\right\} .
$$

Since $0 \in S^{*}$, we observe that $S^{*}$ is nonempty. Also it is easily shown that $S^{*}$ is closed.

* Received by the editors July 21, 1969. Presented by invitation at the Fourth Gatlinburg Symposium on Numerical Algebra supported by the National Science Foundation and sponsored by Society for Industrial and Applied Mathematics, held in Gatlinburg, Tennessee, April 13-18, 1969. This research was supported in part by the National Science Foundation under Grant GP-7073.

† Department of Mathematics, University of Wisconsin, Madison, Wisconsin 53706.

$\ddagger$ Department of Electrical Engineering, Marquette University, Milwaukee, Wisconsin 53233. 
Definition 3. If $C$ is a cone in $R^{n}$ and $x=y-z$ where $y \in C, z \in C^{*}$ and $(z, y)=0$, then $y, z$ will be called an orthogonal decomposition of $x$ on $C$. Where convenient, we shall refer to $x=y-z$ as an orthogonal decomposition on $C$.

Lemma 1 is essentially to be found in [4] for $C$ such that $C^{*} \subseteq C$ and is used in the section of this paper dealing with symmetric matrices.

LEMMA 1. Let $C$ be a cone in $R^{n}$. Then every $x \in R^{n}$ has an orthogonal decomposition on $C$.

Proof. Let $y$ be the vector $C$ whose distance $\|x-y\|$ from $x$ is minimal over all vectors in $C$ (such a $y$ exists, since $C$ is closed) and let $z=y-x$. Let $v \in C$. Then for all $\varepsilon>0,(y+\varepsilon v) \in C$ and so

$$
\|z\|^{2} \leqq\|x-(y+\varepsilon v)\|^{2}=\|z+\varepsilon v\|^{2}=\|z\|^{2}+2 \varepsilon(z, v)+\varepsilon^{2}\|v\|^{2} .
$$

Hence for all $\varepsilon>0,(z, v) \geqq-\varepsilon\|v\|^{2} / 2$ whence $(z, v) \geqq 0$. It follows that $z \in C^{*}$. Next, observe that $(1-\varepsilon) y \in C$ for $0 \leqq \varepsilon \leqq 1$. Hence

$$
\|z\|^{2} \leqq\|x-(1-\varepsilon) y\|^{2}=\|z-\varepsilon y\|^{2}=\|z\|^{2}-2 \varepsilon(z, y)+\varepsilon^{2}\|y\|^{2} ;
$$

so for all $\varepsilon, 0 \leqq \varepsilon \leqq 1,(z, y) \leqq \varepsilon\|y\|^{2} / 2$. Hence $(z, y) \leqq 0$. But $y \in C$ and $z \in C^{*}$ so that $(z, y) \geqq 0$ and so $(z, y)=0$. The lemma is proved.

The decomposition is in fact unique, but we shall make no use of this.

Several well-known results are consequences of Lemma 1. To illustrate this point, we shall give a proof of Lemma 2 (cf. Fenchel [3, p. 10], Ben-Israel [1]), but in the case of Lemmas 3 and 4 we omit the details. We shall denote the (absolute) boundary of a set $S$ by $\partial S$ and its (absolute) interior by $S^{\circ}$.

LeMma 2. Let $C$ be a cone in $R^{n}$. Then $C^{* *}=C$.

Proof. It is clear from the definitions that $C \subseteq C^{* *}$. So let $x \in C^{* *}$, and let $x=y-z$ be its orthogonal decomposition on $C$. Then

$$
(z, x)=(z, y)-(z, z)=-\|z\|^{2} .
$$

But $x \in C^{* *}$ and $z \in C^{*}$ whence $(z, x) \geqq 0$. It follows that $\|z\|^{2}=0$, and so $z=0$. Hence $x=y \in C$. Thus $C^{* *} \subseteq C$, and the result follows.

Lemma 3. Let $C$ be a cone in $R^{n}$, and let $y \in C$. Then there exists a $z \in C^{*}$ such that $(z, y)=0$ if and only if $y \in \partial C$. If $y \neq 0$, any such $z \in \partial C^{*}$.

One half of the lemma is equivalent to the existence of a support plane at any point of the boundary of the cone, and this result may also be found in Fenchel $[3$, p. 8].

Corollary 1 . Let $C$ be a cone in $R^{n}$, and let $y \notin C^{\circ}$. Then there exists $z \in C^{*}$ such that $(z, y) \leqq 0$.

Lemma 4 (Fenchel [3, p. 12]). If $C$ is a cone in $R^{n}$, then so is $C^{*}$.

A result more general than Lemma 4 is given by Lemma 5 . We identify $R^{m n}$ with the space of all real $m \times n$ matrices.

LemMA 5. Let $C$ be a cone in $R^{n}$, and let $D$ be a cone in $R^{m}$. Let $\Gamma(C, D)$ be the set of all matrices $A \in R^{m n}$ such that $A C \subseteq D$. Then $\Gamma(C, D)$ is a cone in $R^{m n}$.

Proof. Properties (i)-(iv) of Definition 1 are easily verified for $\Gamma(C, D)$. Since $C^{*}$ is a cone, and so $C^{*}-C^{*}=R^{n}$, there exists a basis $x_{1}, \cdots, x_{n}$ for $R^{n}$ with $x_{i} \in C^{*}, i=1, \cdots, n$. Similarly, since $D-D=R^{m}$, there is a basis $y_{1}, \cdots, y_{m}$ for $R^{m}$ with $y_{j} \in D, j=1, \cdots, m$. It then follows that $y_{j} x_{i}^{T}, i=1, \cdots, n, j=1, \cdots, m$, 
is a basis for $R^{m n}$. But $y_{j} x_{i}^{T} \in \Gamma(C, D)$, and thus $\Gamma(C, D)$ satisfies condition (v) of Definition 1 for $R^{m n}$. If $A \in \Gamma(C, D) \cap(-\Gamma(C, D))$, then $A C \subseteq D$; and $A C \subseteq-D$ whence $A C=\{0\}$, since $D \cap(-D\}=\{0\}$. Since $C-C=R^{n}$. it follows that $A R^{\prime \prime}=\{0\}$, whence $A=0$. Thus condition (vi) of Definition 1 is satisfied, and $\Gamma(C, D)$ is a cone.

\section{Cross-positive matrices.}

Definition 4. Let $C$ be a cone in $R^{n}$. An $n \times n$ matrix $A$ is called cross-positive on $C$ if for all $y \in C, z \in C^{*}$ such that $(z, y)=0$ we have $(z, A y) \geqq 0$.

Definition 5. Let $C$ be a cone in $R^{n}$. An $n \times n$ matrix $A$ is called strongly cross-positive on $C$ if

(i) $A$ is cross-positive on $C$,

(ii) for each $y \in \partial C, y \neq 0$, there exists $z \in C^{*}$ such that $(z, y)=0$ and $(z, A y)>0$.

Definition 6. Let $C$ be a cone in $R^{n}$. An $n \times n$ matrix $A$ is called strictly cross-positive on $C$ if for all $y \in C, z \in C^{*}, y \neq 0, z \neq 0$ such that $(z, y)=0$, we have $(z, A y)>0$.

Let $C$ be a cone in $R^{n}$, and let $A C \subseteq C$. In [5, Definition 4.1] Vandergraft has given an interesting definition of the irreducibility of $A$ on $C$. He has shown [5, Theorem 4.1 and Lemma 4.2] that each of the following conditions (which also have been considered by other authors) are equivalent to irreducibility as defined by him.

Condition $\mathrm{I}_{1}$. $A$ has no eigenvector in $\partial C$.

Condition $\mathrm{I}_{2} \cdot(I+A)^{n-1}(C \backslash\{0\}) \subseteq C^{\circ}$.

Thus we shall call $A$ irreducible on $C$ if $A C \subseteq C$ and $A$ satisfies either of the equivalent conditions $\mathrm{I}_{1}$ or $\mathrm{I}_{2}$.

The following symbols are introduced for the sake of convenience:

$\Sigma(C)=\{A: A$ is cross-positive on $C\}$,

$\Sigma^{\prime}(C)=\{A: A$ is strongly cross-positive on $C\}$,

$\Sigma^{+}(C)=\{A: A$ is strictly cross-positive on $C\}$,

$\Pi(C)=\{A: A C \subseteq C\}$,

$\Pi^{\prime}(C)=\{A: A$ is irreducible on $C\}$,

$\Pi^{+}(C)=\left\{A: A(C \backslash\{0\}) \subseteq C^{\circ}\right\}$,

$\Pi_{1}(C)=\{A: A+\alpha I \in \Pi(C)$ for some $\alpha \geqq 0\}$

$=\{A: A+\alpha I \in \Pi(C)$ for some real $\alpha\}$,

$\Pi_{1}^{\prime}(C)=\left\{A: A+\alpha I \in \Pi^{\prime}(C)\right.$ for some $\left.\alpha \geqq 0\right\}$

$=\left\{A: A+\alpha I \in \Pi^{\prime}(C)\right.$ for some real $\left.\alpha\right\}$,

$\Pi_{1}^{+}(C)=\left\{A: A+\alpha I \in \Pi^{+}(C)\right.$ for some $\left.\alpha \geqq 0\right\}$

$=\left\{A: A+\alpha I \in \Pi^{+}(C)\right.$ for some real $\left.\alpha\right\}$.

We shall write $\mathrm{cl}(S)$ for the topological closure of a nonempty set $S$.

Lemma 6. Let $C$ be a cone in $R^{n}$. Then in $R^{n n}$,

$$
\operatorname{cl}\left(\Sigma^{+}(C)\right)=\Sigma(C) \text {. }
$$

Proof. It is easily verified from Definition 4 that $\Sigma(C)$ is closed in $R^{n n}$. For $A \in \Sigma(C)$ and $\delta>0$, define

$$
A_{\delta}=A+\delta y z^{T}
$$


where $y \in C^{\circ}$ and $z \in\left(C^{*}\right)^{\circ}$. Then $A_{\delta} \in \Sigma^{+}(C)$ for all $\delta>0$, and $\lim _{\delta \rightarrow 0} A_{\delta}=A$, whence $A \in \mathrm{cl}\left(\Sigma^{+}(C)\right)$. The lemma now follows since $\Sigma^{+}(C) \subseteq \Sigma(C)$.

Lemma 7. Let $C$ be a cone in $R^{n}$ and let $A \in \Sigma^{\prime}(C)$. Then $A$ has no eigentector in $\partial C$.

Proof. Suppose $u \in \partial C$ and $A u=i u$. Since $A \in \Sigma^{\prime}(C)$, there is a $z \in C^{*}$ such that $(z, u)=0$ and $(z, A u)>0$. But $(z, A u)=i(z, u)=0$. This is a contradiction, and the lemma follows.

We postpone until $\S 5$ the fuller results on eigenvectors and eigenvalues.

THEOREM 1. Let $C$ be a cone in $R^{n}$. Then $\Pi^{\prime}(C)=\Pi(C) \cap \Sigma^{\prime}(C)$.

Proof. If $n=1$, the theorem is clearly valid, because every matrix is in $\Sigma^{+}(C)$ and every nonnegative matrix is in $\Pi^{\prime}(C)$. So let $n \geqq 2$. Suppose $A \in \Pi(C) \cap \Sigma^{\prime}(C)$. By Lemma $7, A$ has no eigenvector in $\partial C$ and so $A \in \Pi^{\prime}(C)$ by Condition $I_{1}$.

Conversely, suppose that $A \in \Pi^{\prime}(C)$. Then $A \in \Pi(C)$, and it only remains to show that $A \in \Sigma^{\prime}(C)$. It is sufficient to prove that $B=(A+I) \in \Sigma^{\prime}(C)$. Let $y \in \partial C$, $y \neq 0$, and $(z, y)=0$. As $A \in \Pi^{\prime}(C), B^{n-1} \in \Pi^{+}(C)$, by Condition $\mathrm{I}_{2}$, so that $B^{n-1} y \in C^{\circ}$, and so $\left(z, B^{n-1} y\right)>0$. Since $B \in \Pi(C)$, we have $\left(z, B^{r} y\right) \geqq 0$ for $r=1, \cdots, n-1$. Since $(z, y)=0$, there exists $r, 1 \leqq r \leqq n-1$, such that $\left(z, B^{r} y\right)>0$ and $\left(z, B^{r-1} y\right)=0$. Let $z^{\prime}=\left(B^{T}\right)^{r-1} z$. Then $z^{\prime} \in C^{*},\left(z^{\prime}, y\right)=0$ and $\left(z^{\prime}, B y\right)>0$. So $B \in \Sigma^{\prime}(C)$ and the theorem is proved.

Corollary 2. $\Pi_{1}^{\prime}(C)=\Pi_{1}(C) \cap \Sigma^{\prime}(C)$.

Remark 1. $A \in \Pi(C)$ if and only if $(z, A y) \geqq 0$ for all $y \in C, z \in C^{*}$.

Note that if $(z, A y) \geqq 0$ for all $z \in C^{*}$, then by Lemma $2, A y \in C$.

Remark 2. If $A \in \Sigma(C)$, so is $A+\alpha I$ for all real $\alpha$, and similarly for $A \in \Sigma^{\prime}(C)$ and $A \in \Sigma^{+}(C)$.

Remark 3. From Remarks 1 and 2 and Corollary 2, the containments shown in Table 1 follow easily. (In Table 1 , an arrow $(\rightarrow)$ is used instead of " $\subseteq$ " for convenience.)

TABLE I

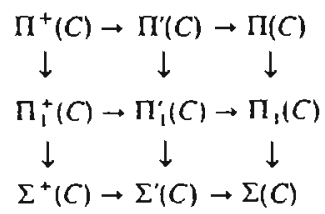

We now investigate the containments $\Sigma^{+}(C) \supseteq \Pi_{1}^{+}(C)$ and $\Sigma(C) \supseteq \Pi_{1}(C)$.

THEOREM 2. $\Sigma^{+}(C)=\Pi_{i}^{+}(C)$ (i.e., a matrix $A$ is strongly cross-positive on a cone $C$ if and only if $(A+\alpha I)(C \backslash\{0\}) \subseteq C^{\circ}$ for some $\left.\alpha\right)$.

Proof. Clearly from Remark 1 and Corollary $1, \Sigma^{+}(C) \supseteq \Pi_{1}^{+}(C)$. Suppose $A \notin \Pi_{1}^{+}(C)$. Then for each real $\alpha, A+\alpha I \notin \Pi^{+}(C)$. So for all $\alpha$ there exists $y_{x} \in C$, $y_{\alpha} \neq 0$, such that $(A+\alpha I) y_{x} \notin C^{\circ}$. Hence by Corollary 1 , there exists $z_{x} \in C^{*}$, $z_{\alpha} \neq 0$, such that $\left(z_{x},(A+\alpha I) y_{\alpha}\right) \leqq 0$. Let $\left\{\alpha_{i}\right\}$ be a sequence of real numbers which approach infinity, and normalize the corresponding $\left\{z_{x_{i}}\right\}$ and $\left\{y_{x_{i}}\right\}$ to unit norm. Then $\left\{z_{x_{i}}\right\}$ and $\left\{y_{x_{i}}\right\}$ have convergent subsequences $\left\{z_{i_{k}}\right\}$ and $\left\{y_{i_{k}}\right\}$ converging to $z$ and $y$ respectively. Let these be renumbered $\left\{z_{i}\right\}$ and $\left\{y_{i}\right\}$, and renumber the corresponding subsequence $\left\{\alpha_{i_{k}}\right\}$ as $\left\{\alpha_{i}\right\}$. Then for all $i,\left(z_{i},\left(A+\alpha_{i} I\right) y_{i}\right) \leqq 0$, whence

$$
\left(z_{i}, A y_{i}\right) \leqq-\alpha_{i}\left(z_{i}, y_{i}\right) \leqq 0 \text {. }
$$


Then, as $i \rightarrow \infty$, we have $z_{i} \rightarrow z, y_{i} \rightarrow y$ and therefore $(z, A y) \leqq 0$. Also

$$
\left(z_{i}, y_{i}\right) \leqq-\frac{1}{x_{i}}\left(z_{i}, A y_{i}\right)
$$

and since $\left(z_{i}, A y_{i}\right)$ is bounded as $i \rightarrow \infty$. it follows that $(z, y) \leqq 0$. But $\left(z, y^{\prime}\right) \geqq 0$ as $z \in C^{*}, y \in C$, so that $(z, y)=0$. Hence there exist $y \in C, z \in C^{*}, y \neq 0, z \neq 0$ such that $(z, y)=0$ and $(z, A y) \leqq 0$. We conclude that $A \notin \Sigma^{+}(C)$. The theorem follows.

Theorem 2 shows that the containment $\Sigma^{+}(C) \supseteq \Pi_{1}^{+}(C)$ is actually an equality. We now show by means of an example that this is false in the case of the containment $\Sigma(C) \supseteq \Pi(C)$. However, as we shall see in $\S 6, \Pi_{1}(C)=\Sigma(C)$ if $C$ is polyhedral.

Example 1. Let $C$ be the circular cone in $R^{3}$ :

$$
C=\left\{x=\left(x_{1}, x_{2}, x_{3}\right)^{T}: x_{1} \geqq 0 \text { and } x_{1}^{2} \geqq x_{2}^{2}+x_{3}^{2}\right\} .
$$

This cone is self polar $\left(C^{*}=C\right)$. Let

$$
A=\left[\begin{array}{lll}
1 & 1 & 0 \\
1 & 1 & 0 \\
0 & 0 & 1
\end{array}\right] .
$$

Suppose $y=\left(y_{1}, y_{2}, y_{3}\right)^{T} \in \partial C$. Then $y_{1} \geqq 0, y_{1}^{2}=y_{2}^{2}+y_{3}^{2}$, and $z \in C,(z, y)=0$ if and only if $z=k\left(y_{1},-y_{2},-y_{3}\right), k \geqq 0$. Hence we have $(z, A y)=k\left(y_{1}^{2}-y_{2}^{2}-y_{3}^{2}\right)$ $=0$ for all $y \in \partial C, z \in \partial C^{*}$ such that $(z, y)=0$ and so $A \in \Sigma(C)$. On the other hand, if $x=(1,0,-1)^{T}$, then $(A+\alpha I) x=(\alpha+1,1,-(\alpha+1))^{T}$ which is not in $C$ for any $\alpha$. It follows that $A \notin \Pi_{1}(C)$. Thus $\Sigma(C)$ contains $\Pi_{1}(C)$ properly.

4. Exponentials of cross-positive matrices. In the case that $C$ is the positive or thant in $R^{n}$. Varga $\left[6\right.$, pp. 257-260] has called $\Pi_{1}(C)$ the class of essentially nonnegative matrices. He has shown that for this cone $C, A \in \Pi_{1}(C)$ if and only if

$$
\exp A=I+A+\frac{1}{2 !} A^{2}+\frac{1}{3 !} A^{3}+\cdots+\epsilon \Pi(C)
$$

For this cone, $\Pi_{1}(C)=\Sigma(C)$ and more generally we have the following theorem.

THEOREM 3. Let $C$ be a cone in $R^{n}$ and let $A$ be a matrix in $R^{n n}$. Then $A \in \Sigma(C)$ if and only if $\exp (t A) \in \Pi(C)$ for all $t \geqq 0$, i.e., $A$ is cross-positive on $C$ if and only if $\exp (t A)$ is positive on $C$ for all $t \geqq 0$.

Proof. Let $A \in \Sigma(C)$. Then by Theorem 2 and Lemma 6, there exist $A_{i} \in \Pi_{1}^{+}(C)$ such that $\lim _{i \rightarrow \infty} A_{i}=A$. Since $A_{i}=B_{i}-\alpha_{i} I$, where $B_{i} \in \Pi(C)$ and $\alpha_{i}$ is real,

$$
\exp \left(t A_{i}\right)=\exp \left(t B_{i}-\alpha_{i} t I\right)=e^{-\alpha_{i} t} \exp \left(t B_{i}\right)
$$

and clearly $\exp \left(t B_{i}\right) \in \Pi(C)$ for all $t \geqq 0$. Hence for all $t \geqq 0$, $\exp \left(t A_{i}\right) \in \Pi(C)$. But $A_{i} \rightarrow \exp \left(t A_{i}\right)$ is a continuous function on $R^{n n}$ for fixed $t$, and $\Pi(C)$ is closed, hence

$$
\exp (t A)=\lim _{i \rightarrow \infty} \exp \left(t A_{i}\right) \in \Pi(C) \quad \text { for all } t \geqq 0 \text {. }
$$
proved)

Conversely, suppose that $\exp (t A) \in \Pi(C)$ for all $t \geqq 0$. Since (as is easily

$$
\lim _{t \rightarrow 0}\left(\frac{1}{t}\right)(\exp (t A)-I)=A \text {, }
$$


and for all positive $t$,

$$
\left(\frac{1}{t}\right)(\exp (t A)-I) \in \Pi_{1}(C)
$$

it follows that $A \in \operatorname{cl}\left(\Pi_{1}(C)\right)=\Sigma(C)$.

Remark 4. It is easily shown that if $\exp (t A) \in \Pi(C)$ for all $t$ in some set $P$ which has accumulation point at $t=0$, then $A \in \Sigma(C)$.

Let $f(z)$ be an analytic function on some domain $D$ in the complex plane. Let $A$ be a complex matrix such that $\operatorname{spectrum}(A) \subseteq D$. If $f^{\prime}(\lambda) \neq 0$ for all $\lambda$ in spectrum $(A)$, then it may be proved by considering the Jordan canonical form of $A$ that

$$
\forall(f(A)-v I)=\Sigma\{\cdot f(A-\lambda I): f(\lambda)=v\},
$$

where $\mathcal{V}^{\prime}(B)$ is the null-space of the matrix $B$. Thus if $f^{\prime}(\lambda) \neq 0$ for all $\lambda$ in $\operatorname{spectrum}(A)$ and $f(\lambda) \neq f(\mu)$, if $\lambda, \mu$ are in $\operatorname{spectrum}(A)$, but $\lambda \neq \mu$, then

$$
\mathscr{N}(f(A)-f(\lambda) I)=\mathscr{N}(A-\lambda I)
$$

for all $\lambda$ in spectrum $(A)$. It follows that under these conditions $A$ and $f(A)$ have the same eigenvectors. We shall apply these remarks to the function $f(z)=e^{i z}$.

Lemma 8. Let $C$ be a cone in $R^{n}$ and let $A$ be an $n \times n$ matrix. Then $\exp (t A)$ $\in \Pi^{\prime}(C)$ for all positive $t$ except possibly on a countable set if and only if

(i) $A \in \Sigma(C)$

and

(ii) $A$ has no eigenvector on $\partial C$.

Proof. Let $\exp (t A) \in \Pi^{\prime}(C)$ for all positive $t$ except possibly on a countable set. Then there exists a sequence $\left\{t_{n}\right\}$ such that $t_{n}>0, \lim _{n \rightarrow \infty} t_{n}=0$, and $\exp \left(t_{n} A\right)$ $\in \Pi^{\prime}(C)$ for all $n$. It follows from Remark 4 that $A \in \Sigma(C)$. To prove (ii), suppose by way of contradiction that $A$ has an eigenvector on $\partial C$, and choose $t>0$ such that $\exp (t A) \in \Pi^{\prime}(C)$. Since every eigenvector of $A$ is also an eigenvector of $\exp (t A)$, $\exp (t A)$ also has an eigenvector on $\partial C$. But this contradicts Condition $I_{1}$ as $\exp (t A) \in \Pi(C)$. Thus (ii) follows.

Now let $A \in \Sigma(C)$ and suppose $A$ has no eigenvector on $\partial C$. From Theorem 3, it follows that $\exp (t A) \in \Pi(C)$ for all $t \geqq 0$. The eigenvalues of $\exp (t A)$ are $\left\{e^{t \mu_{i}}\right.$, $i=1, \cdots, n\}$ where $\left\{\mu_{i}, i=1, \cdots, n\right\}$ are the eigenvalues of $A$. Let $\mu_{k}=\sigma_{k}+i \omega_{k}$, $k=1, \cdots, n$, where $i^{2}=-1$. Let

$$
F=\left\{t: t>0, t=\frac{2 \pi p}{\omega_{j}-\omega_{k}}, p \text { an integer }, \sigma_{j}=\sigma_{k}, \omega_{j} \neq \omega_{k}\right\} .
$$

Clearly $F$ is either empty or countable, and if $t \notin F$, then $e^{i \mu_{j}} \neq e^{i \mu_{k}}$ whenever $\mu_{j} \neq \mu_{\mathrm{k}}$. Hence by the preceding remarks, if $t \notin F$, every eigenvector of $\exp (t A)$ is also an eigenvector of $A$. Since $A$ has no eigenvector in $\partial C$, it follows that for $t \notin F, \exp (t A)$ has no eigenvector $\partial C$. Since $\exp (t A) \in \Pi(C)$ for all $t \geqq 0$, and since $\exp (t A)$ satisfies Condition $I_{1}$ for $t \notin F$, it follows that $\exp (t A) \in \Pi^{\prime}(C)$ for $t \notin F$. The theorem follows.

Remark 5. Let $E=\left\{t: \exp (t A) \in \Pi(C) \backslash \Pi^{\prime}(C)\right\}$. Clearly $E \subseteq F$, and either $E=\varnothing$ or $E$ is infinite. For if $t \in E$, so is $m t \in E$ for all positive integers $m$. 
Theorem 4. Let $C$ be a cone in $R^{n}$, and let $A \in \Sigma^{\prime}(C)$. Then $\exp (t A) \in \Pi^{\prime}(C)$ for all $t>0$, except possibly on a countable set.

Proof. The theorem follows immediately from Lemmas 7 and 8.

Example 2. Let $C$ be the cone of Example 1, and let

$$
4=\left[\begin{array}{rrr}
0 & 0 & 0 \\
0 & 0 & 1 \\
0 & -1 & 0
\end{array}\right] .
$$

Then $A \in \Sigma(C)$ but $A \notin \Sigma^{\prime}(C)$. Further, $A$ has no eigenvector in $\partial C$ and

$$
\exp (t A)=\left[\begin{array}{ccc}
1 & 0 & 0 \\
0 & \cos t & \sin t \\
0 & -\sin t & \cos t
\end{array}\right]
$$

Thus $\exp (t A) \in \Pi^{\prime}(C)$ for all positive $t$, except $t=2 \pi k, k$ an integer. Indeed, $\exp (t A) \in \Pi^{\prime}(C) \backslash \Pi^{+}(C)$ for all such $t$. This example illustrates that the converse of Theorem 4 is false, and also that the exceptional set $E$ may be nonempty.

It is instructive to compare Lemma 7 and Theorem 4 with the following propositions. For the case that $C$ is the positive orthant, their proof is to be found in Varga [6, pp. 257,260] and is essentially the same in the general case.

Proposition 1. If $A \in \Pi_{1}^{\prime}(C)$, then

$$
\exp (t A) \in \Pi^{+}(C) \text { for all } t>0 \text {. }
$$

Proposition 2. If $A \in \Pi_{1}(C) \backslash \Pi_{1}^{\prime}(C)$, then

$$
\exp (t A) \in \Pi(C) \backslash \Pi^{\prime}(C) \text { for all } t \geqq 0 \text {. }
$$

Corollary 3. If $A \in \Pi_{1}(C)$, then

$$
\exp (t A) \in\left(\Pi(C) \backslash \Pi^{\prime}(C)\right) \cup \Pi^{+}(C) \text { for all } t \geqq 0 \text {. }
$$

If $\Sigma(C)=\Pi_{1}(C)$, the converses hold of the above propositions and corollary.

5. Extensions of the Perron-Frobenius theorems. It may be helpful to explain the relation of our theorems to the Perron-Frobenius theory for cones. In view of Theorem $2\left(\Sigma^{+}(C)=\Pi_{1}^{+}(C)\right)$ it is easy to extend the strong Perron-Frobenius Theorem for cones $C$ in $R^{n}$ (Vandergraft [5, Theorems 4.3 and 4.4] et al.) to $\Sigma^{+}(C)$ (Theorem 5). We then use Lemma $6\left(\Sigma(C)=\operatorname{cl}\left(\Sigma^{+}(C)\right)\right.$ ) to obtain a theorem of Perron-Frobenius type for $\Sigma(C)$ (Theorem 6). In the case of $\Sigma^{\prime}(C)$, we use Theorem 4 to derive Theorem 7 .

THEOREM 5. Let $C$ be a cone in $R^{n}$ and let $A \in \Sigma^{+}(C)$. Let

$$
\lambda=\max \{\operatorname{Re} \mu: \mu \in \operatorname{spectrum}(A)\} .
$$

Then

(i) $\lambda$ is a simple eigenvalue of $A$,

(ii) $\hat{\lambda}>\operatorname{Re} \mu$ for any other eigenvalue,

(iii) the unique eigenvector " of $A$ corresponding to $\lambda$ lies in $C^{\circ}$,

(iv) $A$ has no other eigenuector in $C$. 
Proof. By Theorem 1 there is an $x, x \geqq 0$. such that $B=A+x I \in \Pi^{+}(C)$. By the strong Perron-Frobenius Theorem [5], the spectral radius $\rho$ of $B$ is a simple eigenvalue, with unique eigenvector $u$ and $u \in C^{\circ}$. Also $B$ has no other eigenvector in $C$. If $\lambda=\rho-\alpha$, then $i$ satisfies $\left({ }^{*}\right)$ and (i)-(iv) follow immediately.

THEOREM 6. Let $C$ be a cone in $R^{n}$ and let $A \in \Sigma(C)$. If

$$
\lambda=\max \{\operatorname{Re} \mu: \mu \in \operatorname{spectrum}(A)\},
$$

then $\lambda$ is an eigenvalue of $A$ and a corresponding eigenvector lies in $C$.

Proof. Let $A \in \Sigma(C)$, and for $\delta>0$, define

$$
A_{\delta}=A+\delta y z^{r}, \quad y \in C^{\circ} \text { and } z \in\left(C^{*}\right)^{\circ} \text {, }
$$

as in the proof of Lemma 6 . Since $A_{\delta} \in \Sigma^{+}(C)$ for $\delta>0$, we see by Theorem 5 that there exists $u_{\delta} \in C^{\circ}$ (assume $\left\|u_{\delta}\right\|=1$ without loss of generality) such that $A_{\delta} u_{\delta}$ $=\lambda_{\delta} u_{\delta}$ and such that $\lambda_{\delta}$ has the property $\lambda_{\delta}>\operatorname{Re} \mu_{\delta}$ for all eigenvalues $\mu_{\delta}$ of $A_{\delta}$. Let $\delta \rightarrow 0$ through a sequence $\left\{\delta_{i}\right\}$ and let $\left\{u_{i}\right\}$ and $\left\{\lambda_{i}\right\}$ be convergent subsequences of $\left\{u_{\delta_{i}}\right\}$ and $\left\{\lambda_{\delta_{i}}\right\}$ respectively, with $u=\lim u_{i} \neq 0$ and $\dot{\lambda}=\lim \lambda_{i}$. Then $A u=\dot{\lambda} u$, where $u \in C$, and $\lambda \geqq \operatorname{Re} \mu$ for all eigenvalues $\mu$ of $A$, since we can find a sequence $\delta_{j}$ such that $\lim _{j \rightarrow \infty} \mu_{\delta_{j}}=\mu$, where $\mu_{\delta_{j}}$ is an eigenvalue of $A_{\delta_{j}}$.

THEOREM 7. Let $C$ be a cone in $R^{n}$ and let $A \in \Sigma^{\prime}(C)$. If

$$
\lambda=\max \{\operatorname{Re} \mu: \mu \in \operatorname{spectrum}(A)\},
$$

then

(i) $\lambda$ is a simple eigenvalue of $A$,

(ii) the unique eigenvector of $A$ corresponding to $\lambda$ lies in $C^{\circ}$,

(iii) $A$ has no other eigenvalue in $C$.

Proof. We shall first prove (i). It follows from Theorem 6 that $\lambda$ is an eigenvalue of $A$. From Theorem 4, it follows that there is a $t>0$ such that $\exp (t A) \in \Pi^{\prime}(C)$. Also $e^{t \lambda}$ is already the spectral radius of $\exp (t A)$. Suppose by way of contradiction that $\lambda$ is not a simple eigenvalue of $A$. Then $e^{t \lambda}$ is a multiple eigenvalue of $\exp (t A)$, which is a contradiction since the spectral radius of a matrix in $\Pi^{\prime}(C)$ is a simple eigenvalue [5]. Hence (i) follows.

Condition (ii) is a direct consequence of Lemma 8.

To prove (iii), let $t$ again be chosen so that $\exp (t A) \in \Pi^{\prime}(C)$. Then $\exp (t A)$ has no eigenvector in $C$ other than the one corresponding to its spectral radius $\left(e^{i \lambda}\right)$. Since every eigenvector of $A$ is an eigen vector of $\exp (t A), A$ has no eigenvector in $C$ other than the one corresponding to $\lambda$.

The matrix of Example 2 shows that the converse of Theorem 7 is false. For the same cone of Example 1, a symmetric matrix which is also a counterexample to the converse of Theorem 7 is

$$
A=\left[\begin{array}{rrr}
2 & 2 & 2 \\
2 & 1 & -1 \\
2 & -1 & 1
\end{array}\right] .
$$

\section{Polyhedral cones.}

DEFINITION 7. Let $C$ be a cone in $R^{n}$. We call the set $S \subseteq R^{n}$ a set of generators for $C$ if for all $x \in C$ there exist $x_{1}, \cdots, x_{s}$ in $S$ such that $x=\sum_{i=1}^{s} \alpha_{i} x_{i}$, where $\alpha_{i} \geqq 0, i=1, \cdots, s$. 
Definition 8. Let $C$ be a cone in $R^{n}$. Then $C$ is a polyhedral cone if and only if $C$ has a finite set of generators.

By a well-known theorem $C$ is a polyhedral if and only if $C^{*}$ is also polyhedral (Frenchel [3, p. 22]).

We now identify $R^{m n}$ with the space of all real $m \times n$ matrices. For such matrices $A, B$ the inner product $(B, A)$ is then given by $(B, A)=\operatorname{trace}\left(B^{T} A\right)$.

Lemma 9. Let $C$ be a polyhedral cone in $R^{n}$, and $D$ a polyhedral cone in $R^{m}$. Let $\Gamma(C, D)$ be the set of matrices $A$ in $R^{m n}$ such that $A C \subseteq D$. Then $\Gamma(C, D)$ is a polyhedral cone in $R^{m n}$.

Proof. By Lemma 5, $\Gamma(C, D)$ is a cone in $R^{m n}$. Since $C$ is polyhedral, there exist generators $u_{1}, \cdots, u_{s}$ for $C$ in $R^{n}$; and since $D^{*}$ is polyhedral, there exist generators $v_{1}, \cdots, v_{i}$ for $D^{*}$ in $R^{m}$. Clearly, $A \in \Gamma(C, D)$ if and only if $A u_{i} \in D$ for $i=1, \cdots, n$ whence $A \in \Gamma(C, D)$ if and only if $\left(v_{j} u_{i}^{r}, A\right)=\operatorname{trace}\left(u_{i} v_{j}^{r}, A\right)=\left(v_{j}, A u_{i}\right)$ $\geqq 0$ for $i=1, \cdots, s, j=1, \cdots, t$. Hence $\Gamma(C, D)$ is the dual of the polyhedral cone $G$ in $R^{m n}$ generated by $v_{j} u_{i}^{r}, i=1, \cdots, s, j=1, \cdots, t$, and hence is polyhedral. The lemma is proved.

If $C^{*}$ is generated by $x_{1}, \cdots, x_{p}$ in $R^{n}$ and $D$ is generated by $y_{1}, \cdots, y_{q}$ in $R^{m}$, then $y_{j} x_{i}^{r} \in \Gamma(C, D)$. It is tempting to conjecture that the $y_{j} x_{i}^{r}, i=1, \cdots, p$, $j=1, \cdots, q$, generate $\Gamma(C, D)$. But this is false in general. For example, let $C=D$ be the cone in $R^{3}$ generated by $y_{1}=(1,0,1)^{r}, y_{2}=(0,1,1)^{r}, y_{3}=(-1,0,1)^{r}$ and $y_{4}=(0,-1,1)^{r}$. Then $C^{*}=D^{*}$ is generated in $R^{3}$ by $x_{1}=(-1,1,1)^{r}, x_{2}=(-1$, $-1,1)^{r}, x_{3}=(1,-1,1)^{r}, x_{4}=(1,1,1)^{r}$. Then $I \in \Gamma(C, D) \subseteq R^{33}$, but $I$ is not in the cone generated by the $y_{j} x_{i}^{r}, i, j=1,2,3$.

THEOREM 8. Let $C$ be a polyhedral cone in $R^{n}$. Then $\Sigma(C)=\Pi_{1}(C)$.

Proof. By Lemma 9, $\Pi(C)$ is a polyhedral cone in $R^{m n}$, say $\Pi(C)$ is generated by $A_{1}, \cdots, A_{p}$. It follows that $\Pi_{1}(C)$ is the set of all linear combinations of -1 , $A_{1}, \cdots, A_{p}$ with nonnegative coefficients and hence $\Pi_{1}(C)$ is closed (Fenchel [3], Ben-Israel [1]). Hence by Lemma 6 and Theorem 2,

$$
\Sigma(C)=\operatorname{cl}\left(\Sigma^{+}(C)\right)=\operatorname{cl}\left(\Pi_{1}^{+}(C)\right) \subseteq \Pi_{1}(C) .
$$

Since $\Sigma(C) \supseteq \Pi_{1}(C)$, the theorem follows.

THEOREM 9. Let $C$ be a polyhedral cone in $R^{n}$. Then

$$
\Sigma^{\prime}(C)=\Pi_{1}^{\prime}(C)
$$

Proof. By Corollary 2 and Theorem 8,

$$
\Pi_{1}^{\prime}(C)=\Pi_{1}(C) \cap \Sigma^{\prime}(C)=\Sigma(C) \cap \Sigma^{\prime}(C)=\Sigma^{\prime}(C) .
$$

Obviously, and more generally, $\Pi_{1}^{\prime}(C)=\Sigma^{\prime}(C)$ if $\Pi_{1}(C)$ is closed.

7. Symmetric matrices. In this section the results of $\S 5$ are strengthened for the case of symmetric matrices.

THEOREM 10. Let $C$ be a cone in $R^{n}$ and let $A$ be a real symmetric matrix in $\Sigma^{+}(C)$. Let $\lambda$ be the largest eigenvalue of $A$. Then

(i) $\lambda$ is a simple eigenvalue,

(ii) the unique eigenvector $u$ corresponding to $\lambda$ lies in $\left(C \cap C^{*}\right)^{\circ}$,

(iii) " is the only eigenvector of $A$ in $C \cup C^{*}$. 
Proof. It is clear from Definition 6 that if $A \in \Sigma^{+}(C)$, then $A^{T} \in \Sigma^{T}\left(C^{*}\right)$. So if $A$ is symmetric, $A \in \Sigma^{+}(C)$ implies $A \in \Sigma^{+}\left(C^{*}\right)$. Then from Theorem 5 , $i$ is a simple eigenvalue of $A$, and from Theorem 5 and its dual for $C^{*}$. it follows that the unique eigenvector $u$ corresponding to $\lambda$ lies in $C^{\circ} \cap\left(C^{*}\right)=\left(C \cap C^{*}\right)^{\circ}$. Further, $u$ is the only eigenvector in $C$ and in $C^{*}$, whence (iii) follows.

THEOREM 11. Let $C$ be a cone in $R^{n}$. Let $A$ be a real symmetric matrix and suppose $A \in \Sigma^{\prime}(C)$ and $A \in \Sigma^{\prime}\left(C^{*}\right)$. Let $\lambda$ be the largest eigenvalue of $A$. Then the properties (i)-(iii) of Theorem 10 hold.

The proof uses Theorem 7 and is analogous to that of Theorem 10 and is therefore omitted.

THEOREM 12. Let $C$ be a cone in $R^{n}$ and let $A$ be a real symmetric matrix in $\Sigma(C)$. If $\lambda$ is the largest eigenvalue of $A$, then there is a corresponding eigenvector in $C \cap C^{*}$.

This theorem is a consequence of Theorem 10 and Lemma 6. But the following independent proof is of interest.

Proof. Let $\lambda$ be the largest eigenvalue of $A$. Since $A$ is symmetric,

$$
\lambda=\sup \left\{\frac{(v, A v)}{(v, v)}, 0 \neq v \in R^{n}\right\}
$$

and

$$
=\left\{\frac{(v, A v)}{(v, v)}, v \neq 0\right\}
$$

if and only if $A v=\lambda v$. So let $x \neq 0$ and $A x=\lambda x$. By Lemma 3, there is an orthogonal decomposition $x=y-z$ of $x$ on $C$. We shall first show that both $A y=\lambda y$ and $A z=\dot{\lambda} z$. If either $y=0$ or $z=0$, this is obvious. So suppose both $y \neq 0$ and $z \neq 0$. Then since $(z, A y) \geqq 0$,

$$
\begin{aligned}
\lambda=\frac{(x, A x)}{(x, x)} & =\frac{(y, A y)+(z, A z)-2(z, A y)}{(y, y)+(z, z)} \\
& \leqq \frac{(y, A y)+(z, A z)}{(y, y)+(z, z)} \\
& \leqq \max \left\{\frac{(y, A y)}{(y, y)}, \frac{(z, A z)}{(z, z)}\right\}
\end{aligned}
$$

If $i \leqq(y, A y) /(y, y)$, then $i=(y, A y) /(y, y)$ whence $A y=\lambda y$. It then follows from $A x=\lambda x$ that $A z=i z$. If $\lambda \leqq(z, A z) /(z, z)$, the argument is similar.

Since $x \neq 0$, either $y \neq 0$ or $z \neq 0$; say $y \neq 0$. Let $-y=y^{\prime}-z^{\prime}$ be the orthogonal decomposition of $-y$ on $C$. Since $C$ is a cone, $-y \notin C$, whence $z^{\prime} \neq 0$. Also $z^{\prime}=y^{\prime}+y \in C$ whence $z^{\prime} \in C \cap C^{*}$. By the argument of the previous paragraph, $A z^{\prime}=\lambda z^{\prime}$. If $z \neq 0$, the argument is similar and the theorem is proved.

The following example shows that not all eigenvectors corresponding to the largest eigenvalue of a symmetric matrix need lie in $C \cap C^{*}$. 
Example 3. Let $C$ be the cone of Example 1 and let

$$
A=\left[\begin{array}{rrr}
0 & 0 & 0 \\
0 & -1 & 1 \\
0 & 1 & -1
\end{array}\right]
$$

Then $A$ is cross-positive on $C$. Its eigenvalues are $0,0,-2$, and two eigenvectors for 0 are $(1,0,0)^{T} \in C=C \cap C^{*}$ and $(0,1,1)^{T} \notin C$.

8. Tables and open questions. The various containment relations can be conveniently summarized in Tables 2,3 and 4 . A cone $C$ is smooth if for each $y \in \partial C$ there is a unique $z \in \partial C^{*}$ such that $(z, y)=0$. (Note that the polar $C^{*}$ of a smooth

TABLE 2

Polyhedral cones

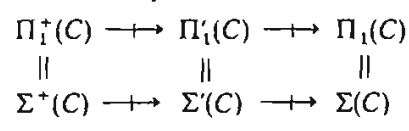

TABLE 3

Smooth cones

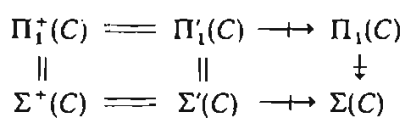

TABLE 4

Generul cones

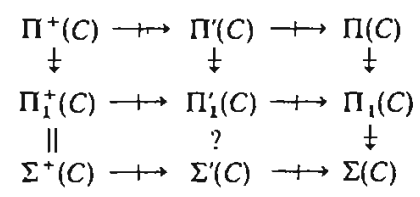

cone $C$ need not be smooth.) For such cones it is obvious from Definitions 5 and 6 that $\Sigma^{+}(C)=\Sigma^{\prime}(C)$, whence also $\Pi_{1}^{+}(C)=\Pi_{1}^{\prime}(C)$ (but in general $\Pi^{+}(C) \subset \Pi^{\prime}(C)$ ).

Tables $2,3,4$ should be read as follows. The symbol $G(C)=H(C)$ means that the sets $G(C)$ and $H(C)$ are equal for all cones $C$ in the class considered. The symbol " $G(C) \rightarrow H(C)$ " means that $G(C)$ is contained in $H(C)$ for all $C$ in the class and that there exists a cone $C$ for which the containment is proper.

The containment relations between the top two rows of Table 4 are omitted from Tables 2 and 3 since they are the same as in Table 4 . The following questions are open.

1. For which cones $C$ in $R^{n}$ is $\Pi_{1}(C)=\Sigma(C)$ ? (Evidently, if and only if $\Pi_{1}(C)$ is closed.)

2. Our main open problem: Is $\Pi_{1}^{\prime}(C)=\Sigma^{\prime}(C)$ for all cones $C$ ? (We know that the equality holds if $\Pi_{1}(C)$ is closed, and therefore if $C$ is polyhedral, and also when $C$ is smooth.) 
3. If $A \in \Sigma^{\prime}(C)$ and $\lambda=\max \{\operatorname{Re} \mu: \mu \in \operatorname{spectrum}(A))$, is $\operatorname{Re} \mu<\lambda$ for $\mu$ $\in \operatorname{spectrum}(A), \mu \neq \lambda$ (cf. Theorem 7)?

4. If $A \in \Sigma^{\prime}(C)$, is $\exp (t A) \in \Pi^{+}(C)$ for all $t>0$ (cf. Theorem 4 and Proposition 1)? Observe that problems 3 and 4 are solved if $\Pi_{1}^{\prime}(C)=\Sigma^{\prime}(C)$.

5. If $A \in \Sigma^{\prime}(C)$, does it follow that $A^{T} \in \Sigma^{\prime}\left(C^{*}\right)$ (cf. Theorem 11)?

\section{REFERENCES}

[1] A. BEN-]SRAEL, Linear equations and inequalities on finite-dimensional real or complex vector spaces, J. Math. Anal. Appl., 27 (1969), pp. 367-389.

[2] G. BIRKHoFf, Linear transformations with invariant cones, Amer. Math. Monthly, 74 (1967), pp. $274-276$.

[3] W. Fenchel, Convex Cones, Sets and Functions, Princeton University Notes, Princeton Univ., Princeton, 1953.

[4] E. HaYnsworth and A. J. Hoffman, Two remarks on copositive matrices, J. Linear Algebra Appl., 2 (1969), pp. 387-392.

[5] G. VAndergraft, Spectral properties of matrices having invariant cones, SIAM J. App], Math., 16 (1968), pp. 1208-1222.

[6] R. S. VARGA, Matrix Iterative Analysis, Prentice-Hall. Englewood Cliffs, New Jersey, 1962.

$[7]$, Nonnegatively posed problems and completely monotonic functions, J. Linear Algebra Appl., 1 (1968), pp. 329-347. 